\title{
Role of ultrasound, Color duplex Doppler and sono-elastography in the evaluation of renal allograft complications
}

\author{
Abeer Mohammed Abd El-Motaal', Rasha M. Dawoud ${ }^{1}$, Mohamed F. Sherif $3^{3 *}$ and Tarek A. Eldiasty ${ }^{4}$
}

\begin{abstract}
Background: Renal transplantation could be considered for patients with end-stage renal disease. Ultrasonography is the imaging method chosen for renal allograft evaluation early in the postoperative period. Sono-elastography is used to estimate tissue stiffness. This study aimed to assess the correlation between sono-elastography and renal allograft histopathology in patients who had transplanted kidney and determine the efficacy of grayscale ultrasound, sonoelastography, and color duplex in evaluation renal allograft complications and correlation with renal function.

Results: Forty patients (26 males and 14 females) who underwent renal transplantation were included. Their ages ranging from 10:52 years; they all subjected to ultrasound, color Doppler, sono-elastography, and histopathology. The studded patients were divided into 3 groups according to histopathology: patient with normal results, patients with ATI, and patients with CAI. The difference between the mean elasticity values between the three groups was statistically highly significant ( $p$ value $<0.001$ ) with high specificity, sensitivity, and accuracy in differentiating ATI and normal groups and also CAI and normal groups, while the lowest sensitivity noticed in differentiating between ATI and CAl groups that is mean elasticity was good to differentiating between ATI and normal groups and also between CAI and normal groups while it was less in differentiating between ATI and CAl groups. As regards the Rl, the highest sensitivity of the RI was in differentiating ATI and normal with high sensitivity, specificity, and accuracy, and the lowest sensitivity, specificity, and accuracy of RI were in differentiating CAl and normal groups.

Conclusion: Transplanted renal allograft could be assessed by combined US, color duplex Doppler, and sonoelastography examination; also we can detect posttransplant complications early. Sono-elastography could be an efficient noninvasive method to diagnose and monitor kidney allograft rejection and follow-up of the renal allograft, which may give a further and possibly earlier prognostic index for chronic dysfunction in addition to serum creatinine.
\end{abstract}

Keywords: Sono-elastography, Renal allograft, Color Duplex Doppler

\section{Background}

Renal transplantation would have to be considered for all patients with end-stage renal disease (ESRD) according to medical and surgical grounds. However, the risk of acute postoperative renal allograft rejection is high, with $40-80 \%$ of patients manifesting oliguria, hyperthermia, water and sodium retention, hypertension, and transplant renal enlargement/hardening/tenderness, ultimately resulting in kidney graft loss $[1,2]$.

\footnotetext{
*Correspondence: msherif1000@yahoo.com

${ }^{3}$ Radiology and Imaging Department, Faculty of Medicine, Tanta University,

Medical Complex, Elgiesh (Elbahr) street, Tanta, Egypt

Full list of author information is available at the end of the article
}

The prognosis may be drastically improved in more than $90 \%$ of patients if the acute rejection is identified and treated at its early stage, but assessment methods are not optimal [3].

Renal transplantation may be followed by many complications. The most frequent complications include perinephric fluid or collections (i.e., hematomas, seromas, urinomas, lymphoceles, and abscesses), decrease renal function (i.e., acute tubular necrosis, rejection and drug nephrotoxicity), abnormalities of the vascular structures (i.e., renal artery and vein stenosis or thrombosis, arteriovenous fistula), urinary tract obstruction, and renal parenchymal disease (i.e., pyelonephritis, infarction, or rejection) [4]. 
Ultrasonography is often the imaging method chosen for transplant evaluation early in the postoperative period, and it can be used for long-term follow-up. It is also used to guide diagnostic and therapeutic interventions, such as biopsy or fluid aspiration when a complication of transplantation develops [4].

B-mode ultrasound is generally used for measuring transplant renal shape, size, hydronephrosis, and perinephric effusion, while color Doppler ultrasound is used to assess transplant renal perfusion. Both methods provide important imaging evidence for identification of renal graft rejection [5].

Color duplex sonography is the easiest, rapid, and noninvasive investigation to evaluate vascular complications and perfusion of the transplanted kidney. It is an excellent method for screening patients suspected to have renal artery stenosis [6].

There is a need for noninvasive methods for completely or partially replacing renal allograft biopsy in the diagnosis of renal allograft fibrosis and chronic allograft dysfunction [7]. One of these attractive alternatives is sono-elastography which is a novel imaging modality that estimates tissue stiffness [6]. Conventional ultrasound approaches fail to reflect renal stiffness after tissue enlargement during acute rejection. In contrast, sono-elastography (SE) provides quantitative information on tissue elasticity distribution for the assessment of renal pathological changes, enabling the indirect evaluation of renal function changes [8].

Sono-elastography is imaging modality that estimates tissue stiffness, especially for superficial organs; it can be further divided as follows: (1) strain elastography, which is also named quantitative and qualitative elastography, including real-time elastography, and (2) shear wave elastography, which is also named quantitative elastography, including transient elastography, acoustic radiation force impulse, and real-time elastography [9].

\section{Aim of work}

This work aims to assess the correlation between sonoelastography and histopathological results of renal allograft in patients who underwent renal transplantation and also determine the efficacy of grayscale ultrasound, sonoelastography, and color duplex in evaluation renal allograft complication and correlation with renal function.

\section{Methods}

This prospective study was carried on 40 patients who underwent renal allograft transplant. The patients were referred to our institute during the period between October 2017 and January 2019. The duration between date of renal transplantation and examination time was ranging between 1 month and 15 years. Ethics committee approval and informed written consent were obtained.
Privacy and confidentiality of all patient data were guaranteed. All data provision was monitored and used for scientific purposes only.

Inclusion criteria:

- The patient underwent renal transplantation who presented with clinical complication, proteinuria, or elevated serum creatinine.

- Patient agrees to participate in the study for healthy subjects included age $\geq 10$ years.

- Patient body mass index less than $35 \mathrm{~kg} \backslash \mathrm{m} 2$.

Exclusion criteria:

- Body mass index greater than $35 \mathrm{~kg} \backslash \mathrm{m} 2$

- Pregnancy or nursing status

- Any conditions that impeded the visualization of the kidney by ultrasound

- Patients who refuse to join this study.

All patients of our study were subjected to the following;

I) Detailed history taking and examination: the patient's history includes personal data and history of presenting complains; any relevant history was assessed.

II) Laboratory investigations according to the patient's condition including creatinine level, calcineurin inhibitors level, complete blood picture, and urine analysis.

III) Ultrasound examination of the abdomen and pelvis (mainly transplanted kidney): -Routine ultrasound, color duplex Doppler, and sono-elastography examination were performed for all patients by using (IU22 Philips medical system, Holland) with a frequency range: $1-5 \mathrm{MHz}$ transabdominal transducer.

The ultrasound technique:

- Patients were placed in the supine position with the abdominal wall over the transplant renal graft side fully exposed. B-mode ultrasound performed to observe transplant renal shape, size, and parenchymal echo, space occupation, collecting system, ureter dilatation, and perinephric effusion.

IV) Color duplex Doppler examination of transplanted kidneys, their main supplying arteries and veins and inferior vena cava:

- Doppler of the kidney graft was repeated within $24 \mathrm{~h}$ in suspected cases of recent transplants 
who have complications or decline of renal function.

Color Doppler ultrasonography was carried out for measurements of:

- Renal parenchymal perfusion

- Peak systolic velocity

- Resistance index(RI) of interlobar arteries (3 values of R.I. were calculated for each renal allograft, and the mean value was taken).

$\mathrm{RI}=$ (peak systolic velocity-end diastolic velocity)/peak systolic velocity

V) Sono-elastography:

- Strain wave elastography was performed for the assessment of parenchymal elasticity for all patients in this study by using a $5-1 \mathrm{MHz}$ trans-abdominal transducer to assess the following:

a. Elasticity value at the upper zone.

b. Elasticity value in the middle zone.

c. Elasticity value at a lower zone.

d. The mean for these measurements was calculated.

- The measurements of kidney stiffness were expressed in terms of Young's modulus (Kpa).

- The results obtained by strain wave sonoelastography will be correlated with those obtained by US and color Duplex Doppler.

VI) Histopathological diagnosis:

- The ultrasound-guided biopsy was obtained from the lateral portion of the most accessible upper/ lower pole of the transplanted kidney using an automatic gun system with 18-gauge true cut needle. In our 40 patients, two cores of each renal allograft tissue were obtained ( 80 cores), and repeated 14 biopsy cores were obtained from $7 \mathrm{pa}$ tients due to insufficient tissue in their biopsy cores (total of 94 biopsy cores). Regarding postallograft biopsy complications of our 40 patients, only mild hematuria occurred in 19 patients that was resolved spontaneously. Small perinephric hematomas (less than $5 \mathrm{~cm}$ ) was seen in 3 renal allografts that was followed up by repeated US examinations that displayed gradual resolving of hematomas during 3 weeks. Histopathological diagnosis of biopsy specimens was obtained and served as reference standards.

- Measurement of strain wave elastography was correlated with histopathological diagnosis.

\section{Results}

Forty patients (26 males (65\%) and 14 females (35\%)) who underwent renal transplantation were included in this study. Their ages ranged from 10 to 52 years (mean age of $32.03 \pm 9.9$ years). The studded patients were divided into 3 groups according to histopathology:

- Patient with normal results

- Patients with ATI

- Patients with CAI

Ultrasound in this study was done for all 40 patients, 3 of them showed minimal peri-graft-free fluid (7.5\%), 2 had simple parenchymal cyst (5\%), 3 was GII echogenicity (7.5\%), 4 showed small stones (10\%), 2 showed perigraft collection (5\%), 1 had heterogeneous collections at pelvis $(2.5 \%)$, and the others were normal (62.5\%).

On correlating perfusion grades with histopathology among our patients, there were 26 patients (65\%) who had good perfusion (Grade 0) where 13 of them showed ATI while 13 showed normal results. Twelve patients (30\%) had reasonable perfusion (Grade 1), and all of them had CAI, and finally, 2 patients (5\%) had patchy perfusion (Grade 2) where 2 patients of them showed normal results and 2 patients had CAI as shown in Table 1.

Regarding histopathological analysis in this study, there were 13 patients with normal renal tissue, 13 patients had ATI, and 14 patients had CAI as shown in Table 2.

The median elasticity value of the normal patients (13 patients) was 5.7 with the range $3.99-10.91$ as shown in Fig. 1, while the median elasticity value of the patients who had ATI (13 patients) was 18.91 with the range 18.24-24.52 as shown in Fig. 2 and in patients who had CAI was 30.62 with the range $30.32-36.90$ as shown in Figs. 3 and 4 and summarized in Table 3.

The statistical analysis as regards the difference between the mean elasticity values in differentiation between the three studied groups in our thesis was highly statistically significant ( $p$ value $<0.001$ ), the specificity and accuracy the same between all groups studied with same sensitivity in differentiating group (ATI and normal and CAI and normal), while the lowest sensitivity at differentiating ATI and CAI that is mean elasticity good

Table 1 Perfusion grades correlating to histopathology in the 3 studied groups

\begin{tabular}{lllll}
\hline & Number of cases $n(\%)$ & Normal $n(\%)$ & ATI $n(\%)$ & CAI $n(\%)$ \\
\hline Grade & & & & \\
0 (good) & $26(65 \%)$ & $13(100)$ & $13(100)$ & 0 \\
1 (reasonable) & $12(30 \%)$ & 0 & 0 & $12(85.7)$ \\
2 (patchy) & $2(5 \%)$ & 0 & 0 & $2(14.3)$ \\
\hline
\end{tabular}


Table 2 Histopathological results of our 40 patients

\begin{tabular}{ll}
\hline Histopathological results & $\begin{array}{l}\text { Number of cases } \\
n(\%)\end{array}$ \\
Normal & $13(32.5 \%)$ \\
ATI & $13(32.5 \%)$ \\
CTI & $14(35 \%)$ \\
\hline
\end{tabular}

to differentiating between (ATI and normal) and (CAI and normal) while less in differentiating between (ATI and CAI) as shown in Table 4.

As regards the mean \pm SD of the resistive index (RI), it was $0.63 \pm 0.06,0.76 \pm 0.03$, and $0.66 \pm 0.05$ in the normal group, ATI group, and CAI group, respectively. There was a statistically significant difference between the normal cases and ATI $\left(p\right.$ value $\left.<0.001^{*}\right)$ as well as between ATI and CAI ( $p$ value $<0.001^{*}$ ), while there is no statistically significant difference between the normal and CRI $p$ value $=0.06$ as shown in Table 5 .

As regards $p$ value $<0.001$, there was statically significant difference between CAI and ATI group and ATI and normal group, while there is no significant difference between group of CAI and normal and the other groups. The table showing the highest sensitivity of the RI was in differentiating ATI and normal with high sensitivity, specificity, and accuracy, and the lowest sensitivity, specificity, and accuracy of the RI were in differentiating CAI and normal as shown in Table 6.
The statistical analysis as regards the correlation between serum creatinine and perfusion $(p=0.16)$ and between serum creatinine and RI $(p=0.94)$ and between serum creatinine and elasticity $(p=0.09)$, showed no statistically significant difference between the serum creatinine and perfusion RI, and elasticity. Also there was no statistically significant difference between the serum calcineurin inhibitors and perfusion, RI, and elasticity.

\section{Discussion}

Kidney transplantation is the treatment of choice for many patients with end-stage renal disease which kidneys are unable to filter wastes from the body, maintain the proper balance of water and chemicals, or produce urine [7].

This study included 40 patients, their ages ranging from 10 to 50 years old and a total mean age $\pm \mathrm{SD}=$ $32.03 \pm 9.9$ years. The female (14) patients representing $35 \%$ and the male patients (26) representing $65 \%$ of the patients match the study published by Ozkan et al., 2013 [10], which included 42 patients with (18) females and (24) males (mostly male) with mean age $38.7 \pm 11.8$ and agreed with Tatar et al. (2014) [11] which included (28) patients with (10) females and 18 males with mean age $39 \pm 11$. So, we can conclude that the incidence of renal failure and renal transplantation is more likely at these age groups with male predominance.

All included patients in this study were examined by ultrasound, color duplex Doppler, and sono-elastography.
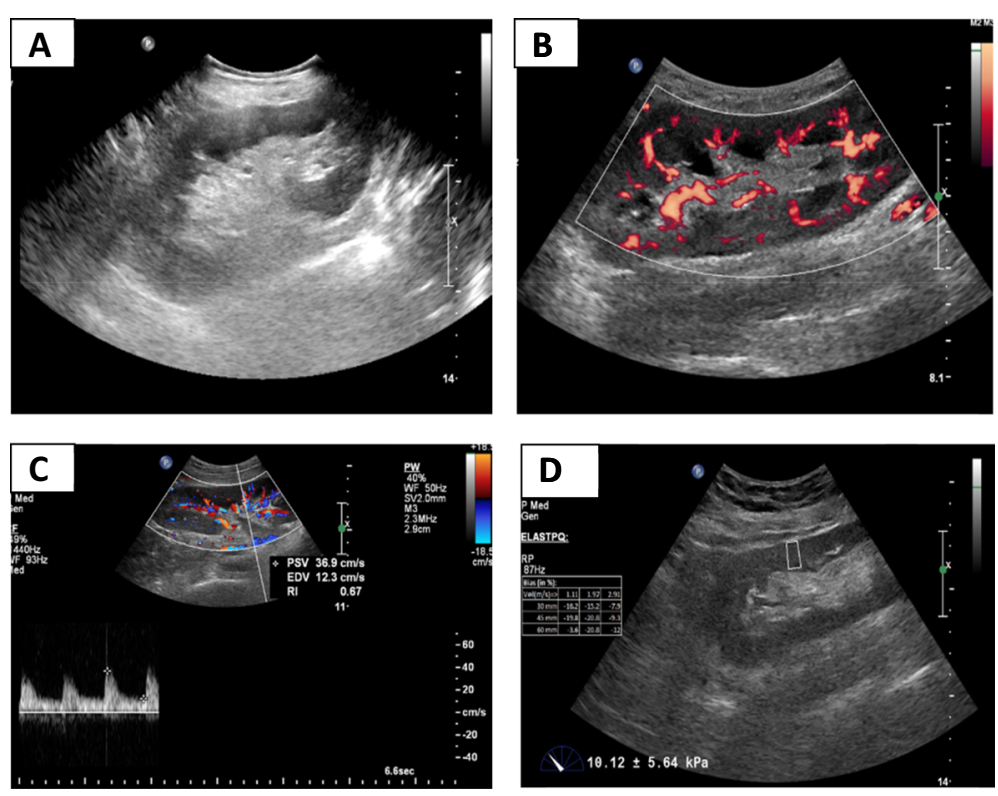

Fig. 1 A 27-year-old male patient who underwent renal transplantation in 2017, on 19 April 2018, was presented with a creatinine level of 2.2 $\mathrm{mg} / \mathrm{dl}$. a Graft US showed average size graft at the right iliac region with normal echo pattern, no back pressure, and no peri-graft collection. b Power Doppler US showed good cortical perfusion. c Duplex Doppler US shows average RI measuring $=0.67$. $\mathbf{d}$ Mean sono-elastography at transplanted kidney measured $10.12+5.64 \mathrm{Kpa}$. Histopathology diagnosis by the US-guided biopsy: normal 

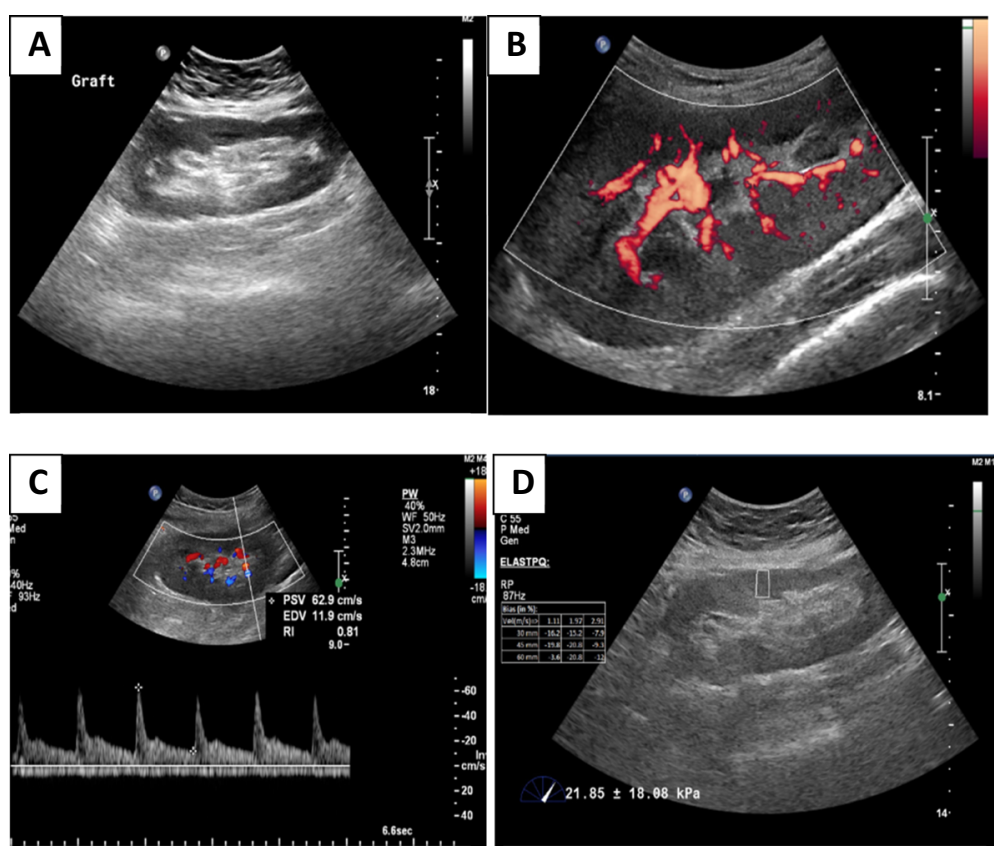

Fig. 2 A 25-year-old female patient who underwent renal transplantation in 2013, during follow-up on 20 November 2017, was presented with creatinine level $1.5 \mathrm{mg} / \mathrm{dl}$ and increase calcineurin inhibitors (CNI). a Graft US showed average size graft at the right iliac region with increased echogenicity, no back pressure, and no peri-graft collection. b Power Doppler US shows good cortical perfusion. c Duplex Doppler US shows elevated RI measuring $=0.81$. d Mean sono-elastography at transplanted kidney measured $21.85+18.08 \mathrm{Kpa}$. Histopathology diagnosis by the US-guided biopsy: acute tubular necrosis (ATI)
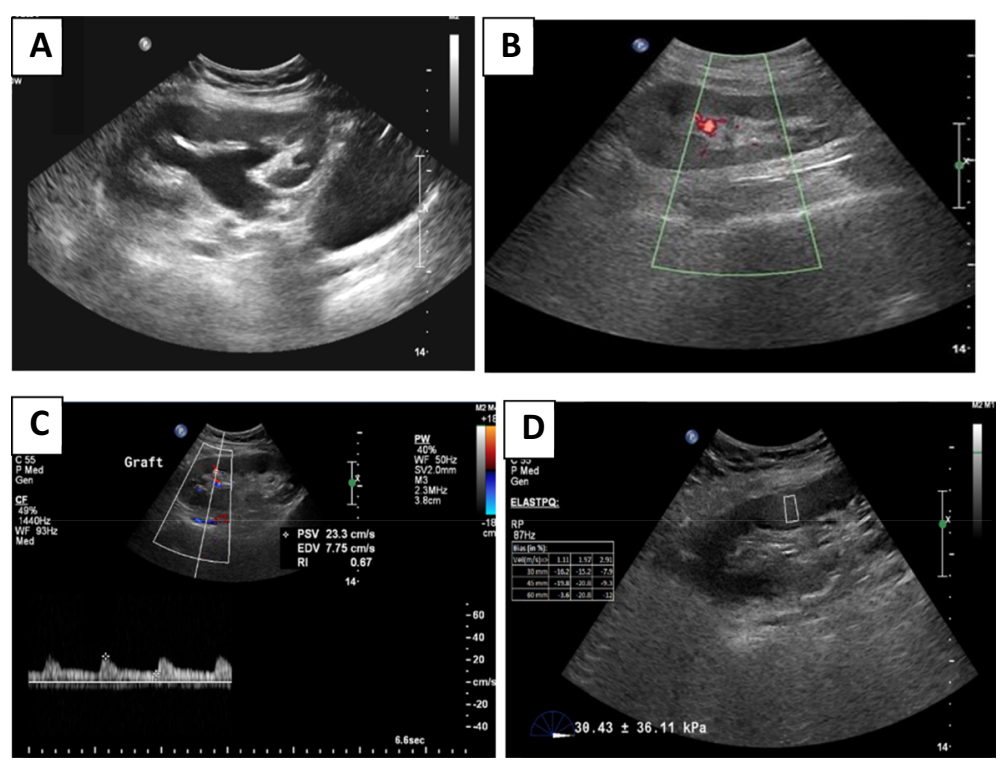

Fig. 3 A 24-year-old female patient who underwent renal transplantation in 2013, during follow-up at 19 December 2017, was presented with creatinine level $1.3 \mathrm{mg} / \mathrm{dl}$ and graft impairment. a Graft US showed average size graft at the right iliac region with normal echo pattern and mild hydronephrosis with full UB, after micturition no hydronephrosis anymore, no peri-graft collection. b Power Doppler US shows patchy cortical perfusion. c Duplex Doppler US showed average Rl $=0.67$. d Mean sono-elastography at transplanted kidney measured $30.43+36.11 \mathrm{Kpa}$. Histopathology diagnosis by the US-guided biopsy: chronic allograft injury (CAI) 

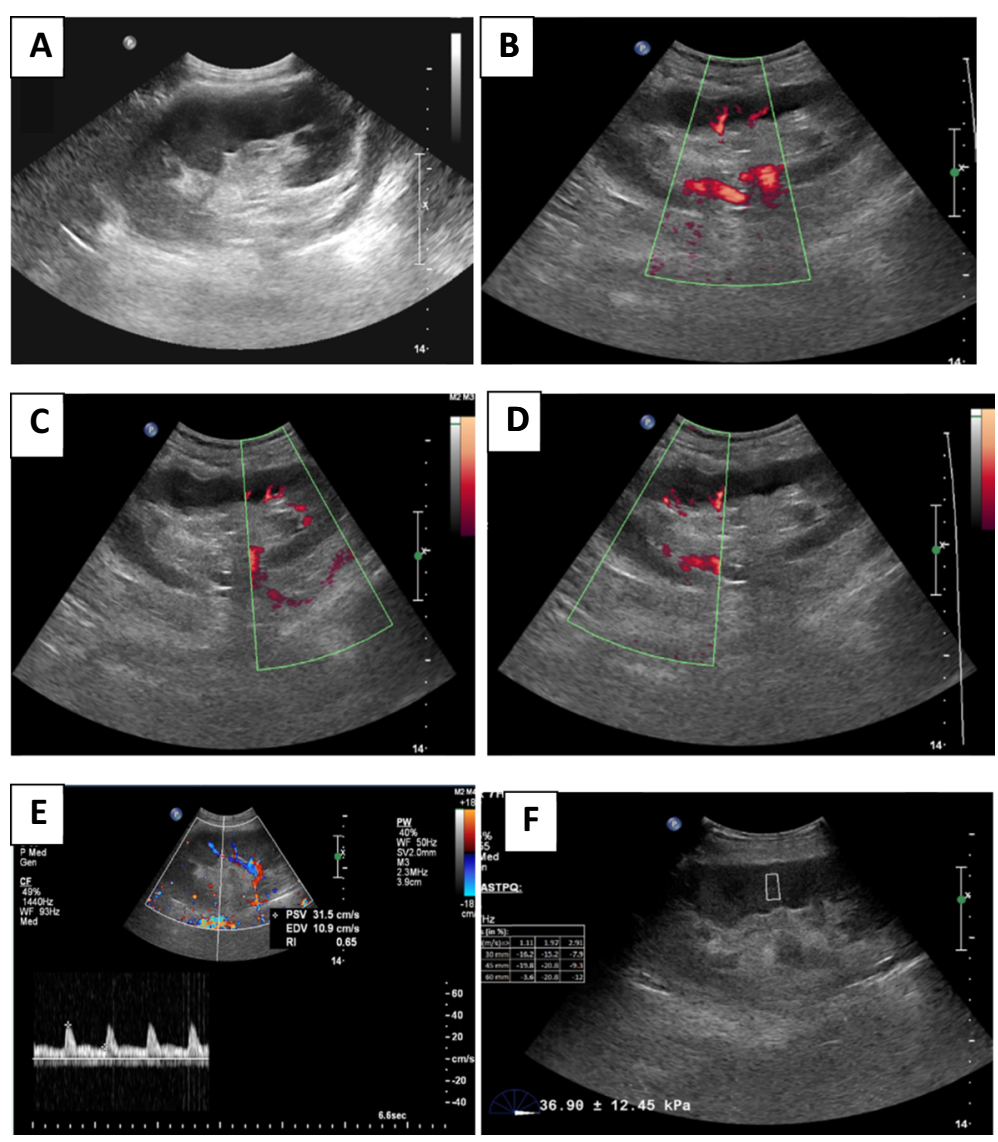

Fig. 4 A 37-year-old male patient who underwent renal transplantation in 2003, during follow-up in 26 March 2018, was presented with his creatinine level $1.8 \mathrm{mg} / \mathrm{dl}$. a Graft the US showed average size graft, normal echo pattern, no back pressure changes, no peri-graft collection. b, c, and d, Power Doppler US showed reasonable cortical perfusion. e, Duplex Doppler US showed average RI $=0.65$. f, Mean sono-elastography at transplanted kidney measured $36.90+12.45 \mathrm{Kpa}$. Histopathology diagnosis by the US-guided biopsy: chronic allograft injury (CAI)

Ultrasonography is often the imaging method chosen for transplant evaluation early in the postoperative period, and it can be used for long-term follow-up as well. It is also used to guide diagnostic and therapeutic interventions, such as biopsy [12]. Color Doppler US has traditionally been the principle noninvasive imaging method for transplant evaluation. It is rapid, safe, portable, and easy to use in unstable postoperative patients [13].

The sono-elastography (SE) provides quantitative information on tissue elasticity distribution for the assessment of renal pathological changes [8].

The final diagnosis of the studied sample was based on histopathology by ultrasound-guided biopsy.
As regards the perfusion grades among our patients and its correlation with histopathological results, we classified our patients on three-grade scale: 13 patients with normal renal tissue and good cortical perfusion (Grade 0), 13 patients with ATI with good cortical perfusion (Grade 0), 14 patients with CAI including 12 reasonable perfusion (Grade 1), and 2 patients with patchy perfusion (Grade 2). Our results matched the results of Hilboen et al. (2014) [14] who studied 18 patients and classified their patients on the same three-grade scale. A study was done by Laszlo et al. (1990) [15] who studied that 9 patients had ATI also used the same perfusion scale.

Table 3 Mean elasticity among studied groups

\begin{tabular}{lllll}
\hline & $\begin{array}{l}\text { Normal median } \\
(\mathrm{IQR})\end{array}$ & $\begin{array}{l}\text { ATI median } \\
(\mathrm{IQR})\end{array}$ & $\begin{array}{l}\text { CAl median } \\
(\mathrm{IQR})\end{array}$ & Test of significance \\
\hline Mean elasticity & 5.7 & 18.91 & 30.62 & $\mathrm{KW}$ \\
& $(3.99-9.91)$ & $(18.24-24.52)$ & $(30.32-36.90)$ & $p<0.001^{*}$ \\
\hline
\end{tabular}


Table 4 Statistic of the means elasticity values between the 3 groups

\begin{tabular}{|c|c|c|c|c|c|c|}
\hline & $\begin{array}{l}\text { AUC } \\
(95 \% \mathrm{Cl})\end{array}$ & $P$ & Cutoff point & $\begin{array}{l}\text { Sensitivity } \\
(\%)\end{array}$ & $\begin{array}{l}\text { Specificity } \\
(\%)\end{array}$ & $\begin{array}{l}\text { Accuracy } \\
(\%)\end{array}$ \\
\hline \multicolumn{7}{|c|}{ Differentiating ATI and normal } \\
\hline Mean elasticity & $\begin{array}{l}1.0 \\
(1.0-1.0)\end{array}$ & $<0.001^{*}$ & 14.96 & 100.0 & 92.3 & 92.6 \\
\hline \multicolumn{7}{|c|}{ Differentiating CAI and normal } \\
\hline Mean elasticity & $\begin{array}{l}1.0 \\
(1.0-1.0)\end{array}$ & $<0.001^{*}$ & 14.96 & 100.0 & 92.3 & 92.6 \\
\hline \multicolumn{7}{|c|}{ Differentiating ATI and CAI } \\
\hline Mean elasticity & $\begin{array}{l}0.97 \\
(0.89-1.04)\end{array}$ & $<0.001^{*}$ & $\geq 26.66$ & 92.9 & 92.3 & 92.6 \\
\hline
\end{tabular}

In between 40 patients who were included in our study, 13 normal patients (32.5\%), 13 ATI patients (32.5\%), and 14 CAI patients (35\%), it means that the incidence of CAI in our study was greater than ATI and normal renal allografts, and this was in agreement with the study done by Yang et al. (2017) [16] which included 50 patients; 15 of them were normal (30\%),15 were ATI (30\%), and 20 were CAI (40\%).

In our study, the mean renal allograft elasticity in normal patients was 3.99-9.91 Kpa, and this was in agreement with Gennisson et al. (2012) [17] who reported that mean elasticity values were $15.4 \pm 2.5$ $\mathrm{Kpa}$ and $10.8 \pm 2.7 \mathrm{Kpa}$, respectively, in abnormal and normal patients. The mean elasticity of CAI range was 30.32-36.90 Kpa which agreed with Brocchi et al. (2014) [18] who stated that the mean renal allograft stiffness value in patients with CRI with stable creatinine was $34.06 \pm 10.72 \mathrm{Kpa}$. The mean elasticity in patients with ATI was 18.24-24.52 Kpa according to our study during the examination.

The mean $\pm \mathrm{SD}$ of resistive index(RI) was $0.63 \pm$ 0.06 in normal group, $0.76 \pm 0.03$ in ATI group, and $0.66 \pm 0.05$ in CAI group; these results matched with the study done by Farina et al. (2007) [19] who studied 3 groups: (A) patient without disease, (B) patient with chronic rejection, and (C) patient with acute tubular necrosis; the range of RI for the Group A was 0.6-0.72, the Group B was 0.69-.79, and the group C was $0.68-78$. On the other hand, our results disagreed with Ozkan et al (2013) [10] who reported that there was no significant correlation between renal parenchymal stiffness and RI in his population, and this is maybe due to the smaller studied group with ATI in his study.
In our study, there is no significant correlation between serum creatinine and elasticity values which agreed with Brocchi et al. (2014) [18] who studied 45 kidney transplant recipients and stated that the mean kidney stiffness value in patients with stable creatinine was $34.06 \pm 10.72 \mathrm{Kpa}$ and no correlation with renal function (single S-creatinine value) was found while disagreed with Yang et al. (2017) [16] who studied 50 patients and stated that there was positive correlation between serum creatinine levels and elastography scores.

In the present study, there is no significant correlation between serum creatinine and perfusion which agreed with Hilboen et al. (2014) [14] who studied 18 patients and stated no significant correlation between the vascular grade perfusion and the mean serum creatinine levels.

In this study, there was no significant correlation between serum creatinine and RI which agree with Hilboen et al. (2014) [14] who studied 18 patients and stated that the percentage correlation between the vascular grades and the RI was $26.4 \%$ ( $p=0.7)$ using person product-moment correlation test, indicating that there was no significant correlation between the vascular grade and the mean RI.

\section{Conclusion}

Transplanted renal allograft could be assessed by combined US, color duplex Doppler, and sono-elastography examination; also we can detect posttransplant complications early. UE could be an efficient noninvasive and inexpensive method to diagnose and monitor kidney allograft rejection and elastographic follow-up of the renal allograft, which may give a further and possibly earlier prognostic index for chronic dysfunction in addition to serum creatinine.

Table 5 Resistive index difference between the three studied groups

\begin{tabular}{|c|c|c|c|c|}
\hline & $\begin{array}{l}\text { Normal Mean } \pm \text { SD } \\
(95 \% \mathrm{Cl})\end{array}$ & $\begin{array}{l}\text { ATI Mean } \pm \text { SD } \\
(95 \% \mathrm{Cl})\end{array}$ & $\begin{array}{l}\text { CAl Mean } \pm \text { SD } \\
(95 \% \mathrm{Cl})\end{array}$ & Test of significance \\
\hline $\mathrm{Rl}$ & $\begin{array}{l}0.63 \pm 0.06 \\
(0.59-0.66)\end{array}$ & $\begin{array}{l}0.76 \pm 0.03 \\
(0.74-0.77)\end{array}$ & $\begin{array}{l}0.66 \pm 0.05 \\
(0.6--0.69)\end{array}$ & $\begin{array}{l}\mathrm{F}=24.6 \\
p=<0.001^{*}\end{array}$ \\
\hline
\end{tabular}


Table $\mathbf{6}$ The statically significant difference of RI between the three studied groups

\begin{tabular}{|c|c|c|c|c|c|c|}
\hline & $\mathrm{AUC}(95 \% \mathrm{Cl})$ & $P$ & Cutoff point & Sensitivity (\%) & Specificity (\%) & Accuracy $(\%)$ \\
\hline \multicolumn{7}{|c|}{ In differentiating ATI Cases and normal } \\
\hline $\mathrm{Rl}$ & $0.97(0.93-1.02)$ & $<0.001^{*}$ & $\geq 0.73$ & 84.6 & 92.3 & 88.5 \\
\hline \multicolumn{7}{|c|}{ In differentiating CAI Cases and normal } \\
\hline $\mathrm{Rl}$ & $0.709(0.51-0.91)$ & 0.06 & $\geq 0.635$ & 71.4 & 61.5 & 66.7 \\
\hline \multicolumn{7}{|c|}{ In differentiating CAI and ATI cases } \\
\hline $\mathrm{Rl}$ & $0.94(0.86-1.02)$ & $<0.001^{*}$ & $\leq 0.73$ & 78.6 & 84.6 & 81.5 \\
\hline
\end{tabular}

\section{Limitation of this study}

Our study showed some limitations. Firstly, in a limited number of patients, in the future, further studies with a higher population are recommended to validate our results.

Secondly, the role of shear wave elastography could not be evaluated as we have used only strain wave elastography.

\section{Abbreviations}

ATI: Acute tubular injuryCAIChronic allograft injuryESRDEnd-stage renal diseaseSE $=$ Sono-elastographyR.I.Resistive index

\section{Acknowledgements}

Not applicable.

\section{Authors' contribution}

All authors have appraised the article and actively contributed in the work. AA contributed in imaging of patients, collecting data, and helping in manuscript writing. RD contributed in the statistical analysis and revision of the manuscript. MS helped in manuscript writing, statistical analysis, and revision of manuscript. TE creates the idea of research and revision of the manuscript. All authors read and approved the final manuscript.

\section{Funding}

The authors declare that the study was self-funded and no funding was obtained from the manufacturers of the machines used, from any of its representatives, or from any other organization.

\section{Availability of data and materials}

The data sets generated and/or analyzed in this study are available through the corresponding author on reasonable request.

\section{Ethics approval and consent to participate}

This study was approved by the Research Ethics Committee of the Faculty of Medicine at Tanta University in Egypt; Reference Number of approval: $31804 / 10 / 17$. All patients or guardians of the patients included in this study gave written informed consent to participate in this research.

\section{Consent for publication}

All parents or guardians of the patients included in this research gave written informed consent to publish the data contained within this study. The authors grant the publisher the consent for publication of this work.

\section{Competing interests}

The authors declare that they have no competing interests.

\section{Author details}

${ }^{1}$ Radio-diagnosis Department, El Mansoura International Hospital, Mansoura, Egypt. ${ }^{2}$ Radio-diagnosis Department, Faculty of Medicine, Tanta University, Tanta, Egypt. ${ }^{3}$ Radiology and Imaging Department, Faculty of Medicine, Tanta University, Medical Complex, Elgiesh (Elbahr) street, Tanta, Egypt. ${ }^{4}$ Radio-diagnosis Department, Mansoura Urology and Nephrology Center, Mansoura, Egypt.
Received: 26 September 2019 Accepted: 13 November 2019 Published online: 16 December 2019

References

1. Knoll G, Cockfield S, Blydt-Hansen T et al (2005) Kidney transplant working group of the Canadian Society of Transplantation. Canadian Society of Transplantation consensus guidelines on eligibility for kidney transplantation. CMAJ 173:1181-1184

2. Andreoni KA, Forbes R, Andreoni RM et al (2013) Age-related kidney transplant outcomes: health disparities amplified in adolescence. JAMA Intern Med 173:1524-1532

3. Venetz JP, Pascual M (2007) New treatments for acute humoral rejection of kidney allografts. Expert Opin Investig Drugs 16:625-633

4. Bron ED, Chen MYN, Wolfman NT, et al(2000): a complication of renal transplantation: evaluation with US and radionuclide imaging. Radio graphics; 20:607-22.

5. Irshad A, Ackerman SJ, Campbell AS et al (2009) an overview of renal transplantation: current practice and use of ultrasound. Semin Ultrasound CT MR 30:298-314

6. De Moris RH, Francisco V, Elias A et al (2003) Duplex Doppler sonography of transplant renal artery stenosis. Journal of clinical ultrasound 31(3):135-141

7. Eisenbrey JR, Dave JK, Forsberg F et al (2016) Recent technological advancements in breast ultrasound. Ultrasonic 70:183-190

8. Grenier N, Gennisson JL, Cornelis F et al (2013) Renal ultrasound elastography. Diagn Interv Imaging 94:545-550

9. Low G, Kruse SA, Lomas DJ (2016) General review of magnetic resonance elastography.World. J Radiol 8:59-72

10. Ozkan F., Yavuz Y.C., Inci M.F., et. al. (2013) Interobserver variability of ultrasound elastography in transplant kidneys: correlations with clinical Doppler parameters. Ultrasound in med.\&Bio;39(1):4-9.

11. Tatar IG, Teber MA, Ogr T et al (2014) Real-time sono-elastographic evaluation of renal allografts in correlation with clinical prognostic parameters: comparison of a linear and convex transducer according to segmental anatomy. Med Ultrason 16:229-235

12. Elizabeth D, Brown MD, Michael Y et al (2000) Complications of renal transplantation: evaluation with US and radionuclide imaging 1 . Radiographic 20:607-622

13. Chiang YJ, Chu SH and Liu KL(2006): Kidney ultrasound is a useful tool in post-transplant follow up. Transplant proc; 38:2018:2019.

14. Hilboen MD, Bude RO, Murphy KJ et al (2014) Renal transplant evaluation with power Doppler sonography. NJR. 70:39-42

15. Laszlo P, Kaveggia Rita R, Perrella Edward G et al (1990) Duplex Doppler sonography in renal allografts: the significance of reversed flow in diastole. AJR 155:295-298

16. Yang J, Wang F, Pan W et al (2017) Correlation between ultrasound elastography parameters and renal function after kidney transplantation. int j. Clin Exp Med 10(2):3211-3217

17. Gennisson JL, Grenier N, Combe C et al (2012) Supersonic shear wave elastography of in vivo pig kidney: influence of blood pressure, urinary pressure, and tissue anisotropy. Ultrasound Med Biol 38:1559-1567

18. Brocchi S, Bortolotti C, Serra C et al (2014) Shear wave elastography in kidney transplantation: a new diagnostic tool to assess chronic allograft fibrosis. https://doi.org/10.1594/ecr2014/C-1199

19. Farina R, Pennisi F, La Rosa M et al (2007) Functional study of the transplanted kidney with power Doppler US and time/intensity curves. Radiol med 112:64-73

\section{Publisher's Note}

Springer Nature remains neutral with regard to jurisdictional claims in published maps and institutional affiliations. 